\title{
Evaluation of selected carotenoids of Lycopersicon esculentum variants as therapeutic targets for 'Alzheimer's disease: an in silico approach
}

Olalekan Olanrewaju Bakare ${ }^{1,2^{*}}$, Adewale Oluwaseun Fadaka ${ }^{1,3}$, Musa Oyebowale Akanbi ${ }^{2}$, Kolajo Adedamola Akinyede ${ }^{4}$, Ashwil Klein ${ }^{5}$ and Marshall Keyster ${ }^{2}$

\begin{abstract}
The seriousness and menace of the worldwide weight of 'Alzheimer's disease have been related to a few factors, which incorporate antioxidant system depletion, mutation of proteins, and high expression of cholinesterases due to aging, environmental influence, diet, infectious agents, and hormonal imbalance. Overexpression of cholinesterases has been emphatically connected to 'Alzheimer's disease because of the unreasonable hydrolysis of acetylcholine and butyrylcholine. Certain plant phytochemicals, for example, beta-carotenoids, lutein, neoxanthin, and viola-xanthine from Lycopersicon esculentum Mill. Var. esculentum (ESC) and Lycopersicon esculentum Mill. Var. cerasiforme (CER) has been utilized altogether as a therapeutic candidate for the treatment of 'Alzheimer's disease. Therefore, this research sought to investigate the drug-likeness of the individual carotenoids as detailed for cholinesterase inhibition in the treatment of 'Alzheimer's disease. Four potential cholinesterase inhibitors from ESC and CER were retrieved from the PubChem database. Investigation of their drug-likeness, toxicity prediction, molecular docking, and dynamic simulations were carried out using Molinspiration, PreADMET V.2.0, Patchdock server, and Schrodinger Maestro software respectively. Neoxanthin was ranked the safest with a greater tendency to inhibit the cholinesterases with high binding affinity. In addition, its stability after simulation in a mimicked biological environment suggests its relevance as a potential drug candidate for the treatment of 'Alzheimer's disease through the inhibition of cholinesterases.
\end{abstract}

Keywords: Toxicity, Drug-likeness, Therapeutic, Inhibitors, Phytochemicals, Ligands, 'Alzheimer's, In silico, Carotenoids

\footnotetext{
* Correspondence: lekanbakare77@gmail.com

${ }^{1}$ Bioinformatics research group, Department of Biotechnology, Faculty of Natural Sciences, University of the Western Cape, Private Bag X17, Bellville, Cape Town 7535, South Africa

${ }^{2}$ Environmental Biotechnology Laboratory (EBL), Department of

Biotechnology, Faculty of Natural Sciences, University of the Western Cape, Cape Town, South Africa

Full list of author information is available at the end of the article
}

(C) The Author(s). 2021 Open Access This article is licensed under a Creative Commons Attribution 4.0 International License, which permits use, sharing, adaptation, distribution and reproduction in any medium or format, as long as you give appropriate credit to the original author(s) and the source, provide a link to the Creative Commons licence, and indicate if changes were made. The images or other third party material in this article are included in the article's Creative Commons licence, unless indicated otherwise in a credit line to the material. If material is not included in the article's Creative Commons licence and your intended use is not permitted by statutory regulation or exceeds the permitted use, you will need to obtain permission directly from the copyright holder. To view a copy of this licence, visit http://creativecommons.org/licenses/by/4.0/ The Creative Commons Public Domain Dedication waiver (http://creativecommons.org/publicdomain/zero/1.0/) applies to the data made available in this article, unless otherwise stated in a credit line to the data. 


\section{Introduction}

Fifty million people have 'Alzheimer's disease worldwide, according to the WHO reports, with approximately ten million cases each year [2]. This disease contributes $70 \%$ of the disability and dependency of older people, thus impacting not only on the psychological, physical, social, but economic aspects of caregivers, families, and societies at large [5]. It is chronic and progressive in nature, causing deterioration of the cognitive function of the brain, either primarily or secondarily, which can affect emotional control, social behaviour, and motivation [13]. However, the recent exponential increase in this disease across ages less than 65 years and associated burden in economically developing countries is becoming alarming [30]. The major contributing factors are that the economically developing countries lack the human resources, adequate screening centres, and ill-equipped to cope with the growing challenges of the disease [40]. Despite enormous efforts by researchers and international communities, the developing pace of the sickness is constantly expanding.

The exact mechanism by which 'Alzheimer's disease affects humans is not fully understood. It is believed that improper folding of tau proteins causing beta-amyloid plaques and neurofibrillary tangle, overproduction of acetylcholinesterase (AChE) and butyrylcholinesterase (BChE), resulting in low stimulation of neurons and depletion of the antioxidant system caused by the 'brain's radical-induced damage due to the low level of antioxidant enzymes and high dependency on oxygen are the major causes of the disease [27]. In the last few years, significant advances have been made regarding the treatment and prevention of this disease [27, 45]. The use of chemotherapy, such as donepezil and galantamine as cholinesterase inhibitors, which hitherto have been used to manage the symptoms of the disease, suffer some limitations such as toxicity, cell cycle specificity, and development of drug resistance [34]. These challenges pose serious threat to the management and cure of the disease, thus developing new molecules as alternative treatment of the disease is imperative.

Three approaches can enhance the treatment of 'Alzheimer's disease, namely: identification of therapeutic candidates from natural sources with inhibitory specificity that will leave the normal brain cells healthy with their development, novel vaccine development for prevention and control, and development of diagnostic tools for early detection [6]. The use of functional foods as $\mathrm{AChE}$ and $\mathrm{BChE}$ has been identified as alternative and cheap sources of therapeutic candidates without hepatotoxicity due to their antioxidant and other compensatory properties. Oboh et al. [35] used two Lycopersicon esculentum varieties extracts as $\mathrm{AChE}$ and $\mathrm{BChE}$ inhibitors for the dietary treatment of 'Alzheimer's disease, where some carotenoids such as lutein, neoxanthin, violaxanthin, and betacarotene, among others were reported to be very abundant in these plant extracts. According to the relative abundance of the GC-MS reports, the four carotenoids mentioned above were reported to be very abundant in these tomato variants. However, it was not stated the extent of these 'carotenoids' drug-likeness in the management of 'Alzheimer's disease.

The current lack of cure for Alzheimer's disease poses a major challenge because many anticholinesterase drugs such as memantine only ameliorate the symptoms of the dementia and are not capable of preventing the progression of the disease $[42,50]$. There is a huge limitation and set-back in the discovery of an effective drug candidate due to the failed clinical trials of the drugs. Attention is currently being focused on natural plant products for the management of the disease due to their low toxicity; while new research innovations are being developed to tailor these phytochemicals into therapeutic agents for neurodegenerative diseases [25, 42]. To this effect, the role of multi-target drug candidates for multifunctional Alzheimer's disease using cholinesterase inhibition and others, for example N-methyl-D-aspartic acid receptor (NMDAR), as molecular targets has been explored [19, 20, 47]; however, several challenges which include undesirable side effects, incessant incidence of drug resistance, and reduced efficacy against diseases for which pathogenesis relies on many biochemical events and bioreceptors working concomitantly necessitate the need for the design of sensitive drugs with specific targets [31].

The use of in silico tools in drug formulations has become increasingly popular because they save time, are less labour intensive, and less expensive with high accuracy and specificity. These tools have been designed to incorporate certain properties which are said to be important for drug formulations. Examples of such properties include solubility, $\mathrm{Ph}$, cytotoxicity, and the screening of impurities or degradants for mutagenicity. These in silico tools include ADMET predictor, Chemicalize online resource, PATCHDOCK, Molinspiration cheminformatics tool, just to mention a few. They are designed to have different functions, which, when used together, can enhance the discovery process by analyzing very large data without worrisome analytical testing. Such functions include a better understanding of the biological responses for the reduction of uncertainties in certain extrapolations and an allowance of prediction of treatment responses that consider 'patients' genetic differences or prior diseases.

Therefore, the aim of this present study was to investigate the drug-likeness and capacity of the four most abundant carotenoids present in two Lycopersicon esculentum varieties for the management of 'Alzheimer's 
disease. These would help proffer a solution to the menace of 'Alzheimer's disease caused by the overexpression and dysregulation of $\mathrm{AChE}$ and $\mathrm{BChE}$, which could subsequently serve as a corrective measure to the degree of alteration and mutation of proteins at the neuromuscular junction. The use of in silico tools would reduce the time and enhance and the quality of the drug discovery. The information gathered from this analysis would guide researchers on the best means to utilize these vital compounds, whether as dietary or from the isolation of each carotenoid compound in the form of a drug for the treatment of the disease.

\section{Methods}

\section{Collection of bioactive compounds}

From our previous study, the four carotenoid compounds which were identified in ESC and CER using GC-MS were further investigated for their inhibitory role against $\mathrm{AChE}$ and $\mathrm{BChE}$ for the treatment of 'Alzheimer's disease [35] with donepezil being used as standard cholinesterase inhibitor [4]. Briefly, beta-carotene, neoxanthin, violaxanthin, and lutein were retrieved from PubChem (https://pubchem.ncbi.nlm.nih.gov/) database in SDF format [26]. Conversion of the SDF files to SMILES files was then carried out using Open Babel Converter (http://openbabel.org/wiki/Main_Page).

\section{Lipinski's rule of five investigation of the bioactive compounds}

The 'Lipinski's rule of five was used to evaluate the 'compounds' drug-likeness with the aid of Molinspiration cheminformatics tool (https://www. molinspiration.com/cgi-bin/properties) that employs a supercomputing resource for biological computation bioinformatics analysis by calculating molecular properties, predicting biological activities, and virtual screening of the ligands [29].

\section{Prediction of toxicity}

The toxicity analysis of the compounds was carried out using preADMET version 2.0 (https://preadmet.bmdrc. $\mathrm{kr} /$ preadmet-pc-version-2-0/) to predict their absorption, distribution, metabolism and elimination/excretion (ADME) properties and evaluate safety through in silico approach [28].

\section{Preparation of ligands and receptors}

LigPrep (https://www.schrodinger.com/products/ligprep) was used to prepare the ligands for further docking analytical studies using Schrodinger Maestro which employs many force fields [36]. The generation of a solitary, reduced energy, and 3D structure with chiralities by optimizing geometries using Desalt, ring conformation, stereochemistries and tautomers, an indication of fruitful structure. Selection of a binding site in the target proteins was carried out prior to the commencement of molecular docking. A method by Fadaka et al. [16] was used to incorporate hydrogen atoms, eliminate alternate conformations and HetAtoms from proteins, add and correct the missing residues, and many more.

\section{Docking studies using PatchDock and Schrodinger maestro software}

The docking analysis of the ligands and the receptors were carried out using two docking tools (Patchdock (https://bioinfo3d.cs.tau.ac.il/PatchDock/php.php) [44] and Schrodinger suit) in order to ensure accuracy according to our previous methods [18, 37]. Briefly, Patchdock server was used to evaluate the atomic contact energy, area, and the binding scores of the complexes while Schrödinger suit was employed to evaluate the binding interaction, dock score, as well as the Glide scores of the ligands against $\mathrm{BChE}$ and $\mathrm{AChE}$. Visualization of the complexes was achieved by Discovery Studio Visualizer (DSV v.4.0).

\section{Calculation of the prime MM-GBSA}

The receptor-ligand complex free binding energy was calculated using the molecular mechanically generalized Born surface area (MM-GBSA) [15, 24]. The binding free energy, relative distance between the complex and PIC50 values were generated.

\section{Molecular dynamics (MD) simulation of the best ligand candidate and the receptors}

MD simulation was carried out using the Desmond module of Schrodinger programming with force field OPLS 2005 [14, 36]. Electrostatic interactions were determined, which computes the 'ligand's relative stability in the binding pockets of the receptors using RMSD plots for both the proteins and the ligand-bound proteins. The analysis of the results was visualized by the simulation interaction diagram and MS-MD trajectory analysis $[1,17]$.

\section{Results}

\section{Retrieval of bioactive compounds}

The four bioactive compounds of ESC and CER reported in our previous work [35] belonged to the carotenoid class of phytochemicals. Their chemical structures were retrieved from the PubChem database with a cholinesterase inhibitor, donepezil, retrieved as control (Table 1). The four bioactive compounds were obtained in SDF formats and were later converted to SMILES formats. The 3-D structures of the PDB files were visualized using BIOVIA Discovery Studio Visualizer (DSV) version 4.0 [3]. 
Table 1 Bioactive Carotenoids of ESC and CER

\begin{tabular}{lllll}
\hline $\mathbf{S} / \mathbf{N}$ & $\begin{array}{l}\text { Name } \\
\text { of } \\
\text { Compo } \\
\text { und }\end{array}$ & $\begin{array}{l}\text { Compound } \\
\text { ID }\end{array}$ & $\begin{array}{l}\text { Molecular } \\
\text { Formula }\end{array}$ & SMILES \\
\hline $\mathbf{1}$ & $\begin{array}{l}\text { Neoxant } \\
\text { hine }\end{array}$ & $\begin{array}{l}\text { PubChem ID: } \\
\text { CID 5282217 }\end{array}$ & $\mathrm{C}_{40} \mathrm{H}_{56} \mathrm{O}_{4}$ & $\mathrm{CC}(=\mathrm{CC}=\mathrm{CC}=\mathrm{C}(\mathrm{C}) \mathrm{C}=\mathrm{CC}=\mathrm{C}(\mathrm{C}) \mathrm{C}=$ \\
& & & $\mathrm{C}=\mathrm{C} 1 \mathrm{C}(\mathrm{CC}(\mathrm{CC} 1(\mathrm{C}) \mathrm{O}) \mathrm{O})(\mathrm{C}) \mathrm{C}) \mathrm{C}=$ \\
& & & $\mathrm{CC}=\mathrm{C}(\mathrm{C}) \mathrm{C}=\mathrm{CC} 23 \mathrm{C}(\mathrm{CC}(\mathrm{CC} 2(\mathrm{O}) \mathrm{C}$
\end{tabular}

\section{Lipinski's rule of five (RO5) analysis}

This analysis, also called 'Pfizer's rule, is used to investigate the drug-likeness of any chemical compound by determining whether the compound has a particular biological attribute or pharmacological relevance that would make it potential oral drug in humans [29]. Another importance of this analysis is the tendency to predict the possibility of success or failure of drug-likeness of molecules simply by fulfilling certain rules such as high lipophilicity depicted as LogP usually below 5, molecular mass not greater than $500 \mathrm{Da}$, hydrogen bond acceptors below 10, hydrogen bond donors below 5, and molar refractivity within the range of 40-130 [23, 29]. Table 2 shows that the four carotenoids did not satisfy all the five requirements, for instance, none of the ligands satisfied the molecular mass which should not be greater $500 \mathrm{Da}$ except donepezil and the ligands also had high lipophilicity greater than 5. Several mechanisms exist to efficiently impact the candidacy of drugs with high molecular weight and high lipophilicity for their proper delivery and bioavailability as oral drug candidates such as nanocomminution $[8,10]$ and high molecular weight drugs have been associated with good stability when compared to low molecular grade polymers [7]. The ligands possessed certain compensatory outcomes for hydrogen bond donors, hydrogen bond acceptors which impact on their stability, properties, and molecular functions [52] whilst excellent molar refractivity values are necessary for the efficient control of dispersive forces in drug-receptor interaction [39]. Interestingly, donepezil satisfied all the rules except very low result for molar refractivity.

\section{Toxicity prediction of the bioactive compounds}

Table 3 reveals neoxanthin, violaxanthin, betacarotene, lutein, and donepezil to be non-carcinogenic and they posed a medium risk in their ability to inhibit HERG. This result indicates varying genetic expression in their

Table 2 Evaluation of the Drug-likeness of the compounds using Lipinski Rule of Five

\begin{tabular}{lllllll}
\hline S/N & Compounds & Molecular Weight (Da) & Hydrogen bond donors & Hydrogen bond acceptors & milogP Values & Molar Refractivity \\
\hline 1 & Neoxanthine & 600,88 & 3 & 4 & 8.70 & 73.21 \\
2 & Violaxanthine & 600.88 & 2 & 4 & 8.99 & 65.51 \\
3 & Betacarotene & 536.89 & 0 & 0 & 9.84 & 40 \\
4 & Lutein & 568.89 & 2 & 2 & 9.31 & 42 \\
5 & Donepezil & 379.50 & 0 & 4 & 4.10 & 38.78 \\
\hline
\end{tabular}


Table 3 Toxicity Prediction results of the bioactive compounds using PreADMET Web server

\begin{tabular}{|c|c|c|c|c|c|c|c|c|c|}
\hline $\mathrm{S} / \mathrm{N}$ & Compounds & Carcinogenic test (Mouse) & HERG Inhibition & BBB & PWS (mg/L) & SP & HIA & PPB & BS \\
\hline 1 & Neoxanthine & Negative & Medium risk & 9.010 & 0.020 & -0796 & 95.175 & 92.807 & 0.0549 \\
\hline 2 & Violaxanthine & Negative & Medium risk & 9.300 & 0.007 & -0.806 & 96.428 & 91.271 & 0.918 \\
\hline 3 & Betacarotene & Negative & Medium risk & 25.995 & 2.646 & -0.609 & 100.000 & 100.000 & 3.363 \\
\hline 4 & Lutein & Negative & Medium risk & 16.260 & 0.003 & -0.648 & 95.521 & 100.000 & 0.002 \\
\hline 5 & Donepezil & Negative & Medium risk & 0.188 & 6.236 & -3.042 & 97.951 & 84.616 & 5.101 \\
\hline
\end{tabular}

Legend: BBB Blood Brain Barrier partition coefficient, PWS Pure Water Solubility, SP Skin Permeability, HIA Human Intestinal Absorption, PPB Plasma Protein Binding, and BS Buffer Solubility

ability to be non-carcinogenic [38]. This indicates the safety of the ligands for consumption. Biological/ pharmacological activities of the five carotenoids were further computed where the blood brain barrier partition coefficient result revealed that beta-carotene had the highest value (25.995) whereas donepezil had the lowest (0.188). Donepezil had the highest pure water solubility property $(6.236 \mathrm{mg} / \mathrm{L})$ with lutein having the lowest value (0.003). All the carotenoids had significant skin permeability values except for donepezil (-3.042); the carotenoids also had significant human intestinal absorption values with beta-carotene having the highest (100.000). All the carotenoids had high plasma protein binding values with donepezil scoring lowest (84.616). The tendency for buffer solubility also revealed lutein with the lowest value of 0.002 .

\section{Molecular docking of the receptors and the ligands}

3-D structure prediction interaction analysis was carried out using the Patchdock server. The binding scores, interface area, and atomic contact energy (ACE) of the complexes were displayed in Table 4 . Patchdock produced higher binding scores for all the ligands than donepezil against $\mathrm{AChE}$ and $\mathrm{BChE}$ with beta-carotene having the highest binding score $(\mathrm{AChE}=6626, \mathrm{BChE}=6548)$ which also ranked highest in interface area $(\mathrm{AChE}=856.60, \mathrm{BChE}=957.20)$

Table 4 Patchdock Analysis of Acetylcholinesterase (AChE) and Butyrylcholinesterase (BChE) and the Carotenoids

\begin{tabular}{lllll}
\hline S/N & Compounds & Binding score & Area & ACE \\
\hline 1 & Neoxanthine- AChE & 6196 & 763.00 & -116.24 \\
2 & Violaxanthine-AChE & 5660 & 686.90 & -85.62 \\
3 & Betacarotene- AChE & 6626 & 856.60 & -266.95 \\
4 & Lutein- AChE & 6502 & 784.40 & -226.07 \\
5 & Donepezil-AChE & 5536 & 646.60 & -202.36 \\
6 & Neoxanthine-BChE & 6086 & 701.00 & -338.06 \\
7 & Violaxanthine- BChE & 6216 & 770.60 & -387.56 \\
8 & Betacarotene- BChE & 6548 & 957.20 & -497.92 \\
9 & Lutein- BChE & 6512 & 807.60 & -461.72 \\
10 & Donepezil- BChE & 5186 & 629.70 & -212.23 \\
\hline
\end{tabular}

and lowest atomic contact energy (ACE) $(\mathrm{AChE}=-$ 266.95, $\mathrm{BChE}=-497.92$ ) against both $\mathrm{AChE}$ and BChE. However, further interaction analysis was required as Patchdock results only showed the interaction between receptors and ligands but not the degree of stability in the receptors [12].

The generated grid box and the ligand structure were used for docking analysis of the receptors $\mathrm{BChE}$ and AChE after optimizing the ligands utilizing the glide module in the Schrodinger suit. Table 5 showed neoxanthin having the best docking scores with binding scores of -9.1 and -10.2 against $\mathrm{BChE}$ and $\mathrm{AChE}$ respectively. No interaction was reported for Betacarotene and lutein against $\mathrm{AChE}$ with violaxanthin having no interaction for BChE. Only donepezil had higher energy than neoxanthin against $\mathrm{AChE}$ and BChE. Neoxanthin formed three stable hydrogen bonding with three amino acids in both AChE (Asp_74, Tyr_72, and His 447) and BChE (Ile_69, Ser_72, and Tyr_332) (Fig. 2), whereas donepezil only formed three stable hydrogen bonds with AChE but none with BChE. The tendency of neoxanthin to form more stable hydrogen bonds with the receptors would impact the stability and molecular functions better than other ligands and donepezil [52].

Also shown in Fig. 1 below is the 2D interaction analysis of the ligands and donepezil with the receptors with all complexes displayed forming non-covalent bonding, an important consideration for drug development and discovery. Higher binding affinity could be observed for neoxanthin against the receptors with violaxanthin and lutein having no interaction with $\mathrm{AChE}$ and $\mathrm{BChE}$, respectively.

Neoxanthin also formed higher hydrogen bonding with AChE (Asp74, Tyr72, and His447) and BChE (Ile69, Ser72, and Tyr332) with relative distances as displayed in Fig. 2. Hence, neoxanthin was therefore selected for molecular dynamics simulation since it has proven more effective in its interaction with the cholinesterases with better stability than donepezil.

\section{Molecular dynamic simulation}

The MD simulation was carried out for neoxanthin against the receptors being the ligand with the best 
Table 5 Binding affinities $(\mathrm{KJ} / \mathrm{mol})$ of the ligands against acetylcholinesterase and butyrylcholinesterase receptors calculated with Glide G score, Dock score, and MM/GBSA

\begin{tabular}{|c|c|c|c|c|c|c|c|c|c|}
\hline \multirow[b]{2}{*}{$\mathrm{S} / \mathrm{N}$} & \multirow[b]{2}{*}{ Compound } & \multicolumn{4}{|c|}{ Acetylcholinesterase (AChE) } & \multicolumn{4}{|c|}{ Butyrylcholinesterase (BChE) } \\
\hline & & Glide score & DOCK SCORE & MM-GBSA & H-Bond & Glide score & DOCK SCORE & MM-GBSA & H-Bond \\
\hline 1 & Donepezil & -16.6 & -16.6 & -81.35 & 3 & -8.9 & -8.9 & -50.25 & 0 \\
\hline 2 & B-carotene & * & * & * & 1 & -5.3 & -5.3 & -30.24 & 1 \\
\hline 3 & Neoxanthin & -10.2 & -10.2 & -103.11 & 3 & -9.1 & -9.1 & -95.11 & 3 \\
\hline 4 & Violaxanthin & -9.1 & -9.1 & -103.43 & 1 & * & * & * & * \\
\hline 5 & Lutein & * & * & * & * & -5.2 & -5.2 & -83.23 & 1 \\
\hline
\end{tabular}

inhibitory activity and was monitored for $100 \mathrm{nsec}$ utilizing four interactions namely; hydrogen bonds, hydrophobic interactions, ionic bonds, and salt bridges (Figs. 3, 4, 5, and 6). In Fig. 3, the neoxanthine root mean square deviation (RMSD) plot indicates the stability with respect to the proteins and its binding pockets throughout the entire period. The 'Lig fit Prot' shows the RMSD of neoxanthin aligned on AChE and BChE backbones. Since the 'Lig fit Lig' showed the RMSD of neoxanthin was lower than the $\mathrm{AChE}$ and $\mathrm{BChE}$ significantly, then it means the neoxanthin had not diffused away from its initial binding sites.
Figure 4 below showed the interaction of neoxanthin with $\mathrm{AChE}(\mathrm{A})$ and $\mathrm{BChE}(\mathrm{B})$ where the interaction residues were depicted in green colour and the receptor secondary structures in terms of helices and $\beta$-strands are indicated with orange and blue bands.

In Fig. 5 below, neoxanthin displayed favoured water bridge interaction with the nitrogen atoms of the amino acids for AChE (A) at Tyr_72, Asp_74, Thr_75, Gly_82, Trp_86, Tyr_124, His_284, Glu_285, Glu_292, and His_ 447 whilst neoxanthin-BChE complex (B) had favoured water bridge interaction at Asn_68, Ile_69, Asp_70, Gln 71, Ser_72, Gly_78, Ser_79, Trp_82, Leu_274, Ala_277, Ala_328, Phe_329, and Tyr_332. Hydrogen bonding

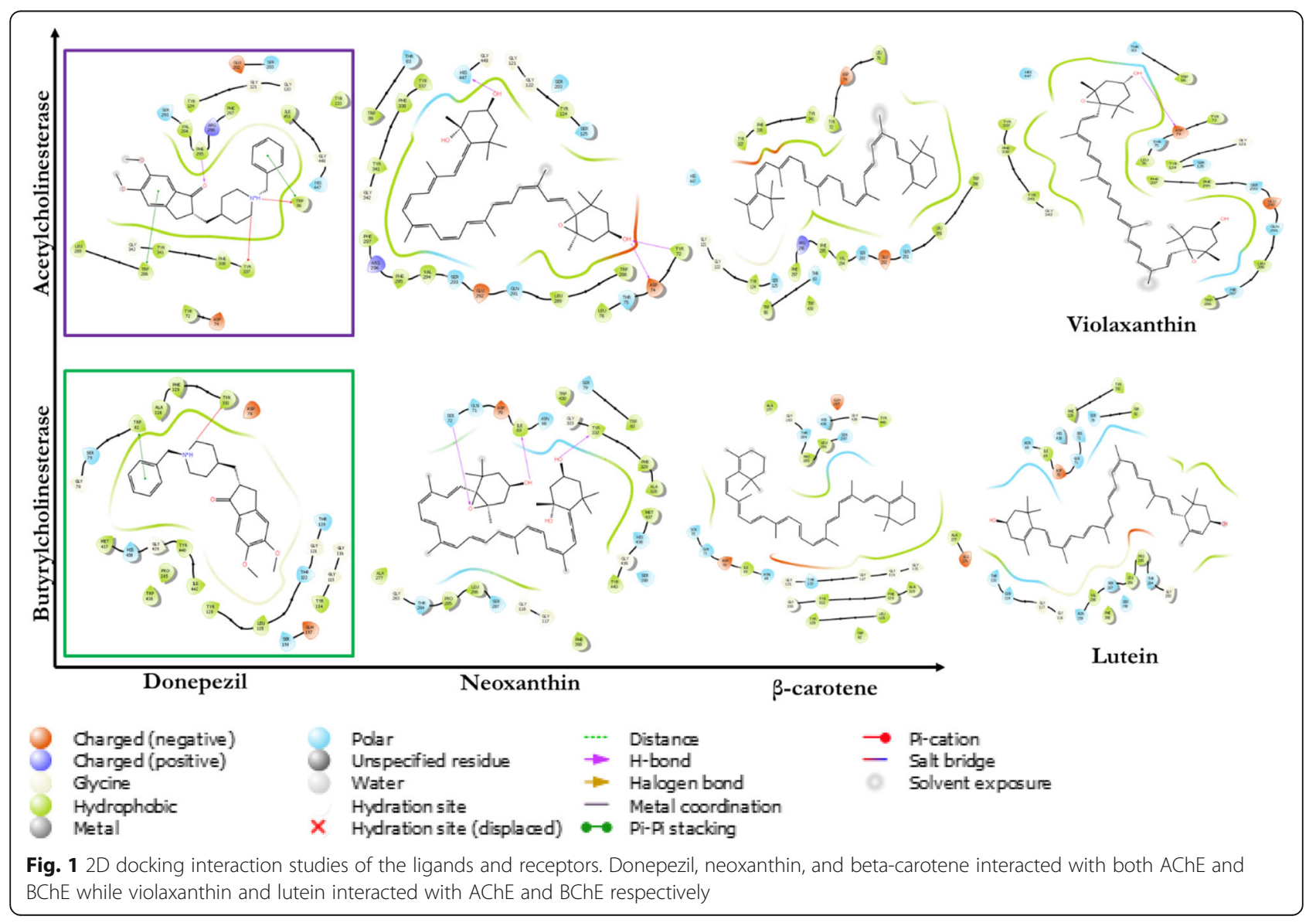




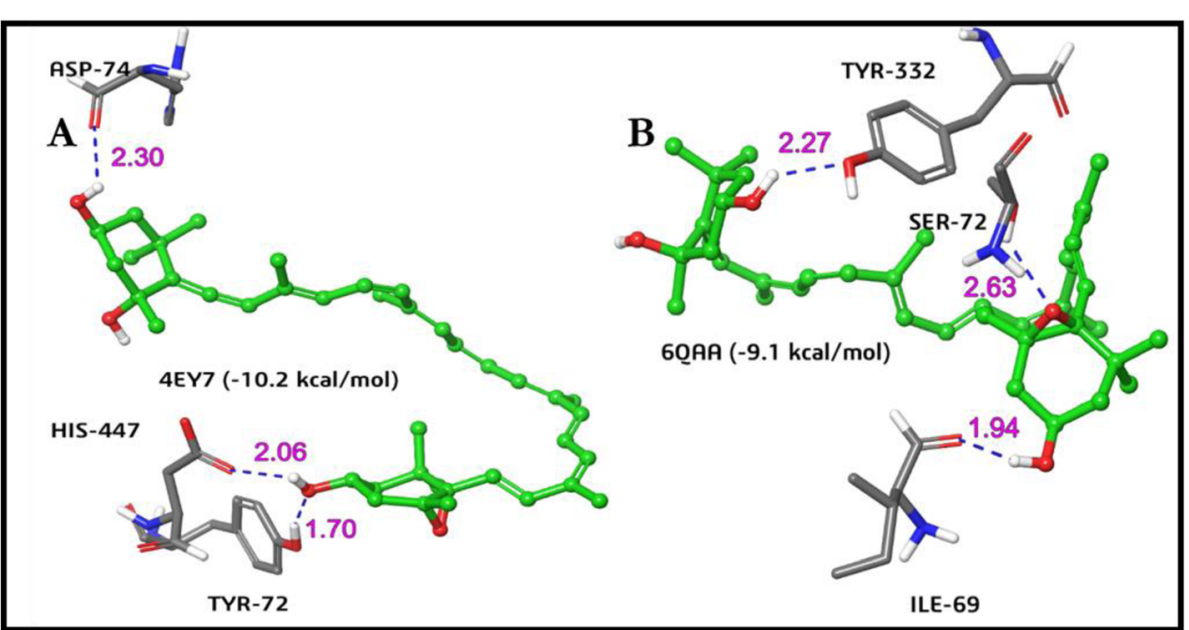

Fig. 2 The docking analysis of Neoxanthin in the active sites of $\mathbf{A}$ acetylcholinesterase and $\mathbf{B}$ butyrylcholinesterase. Hydrogen bonds are represented with blue dotted line with their respective bond distance (pink) in $\AA$

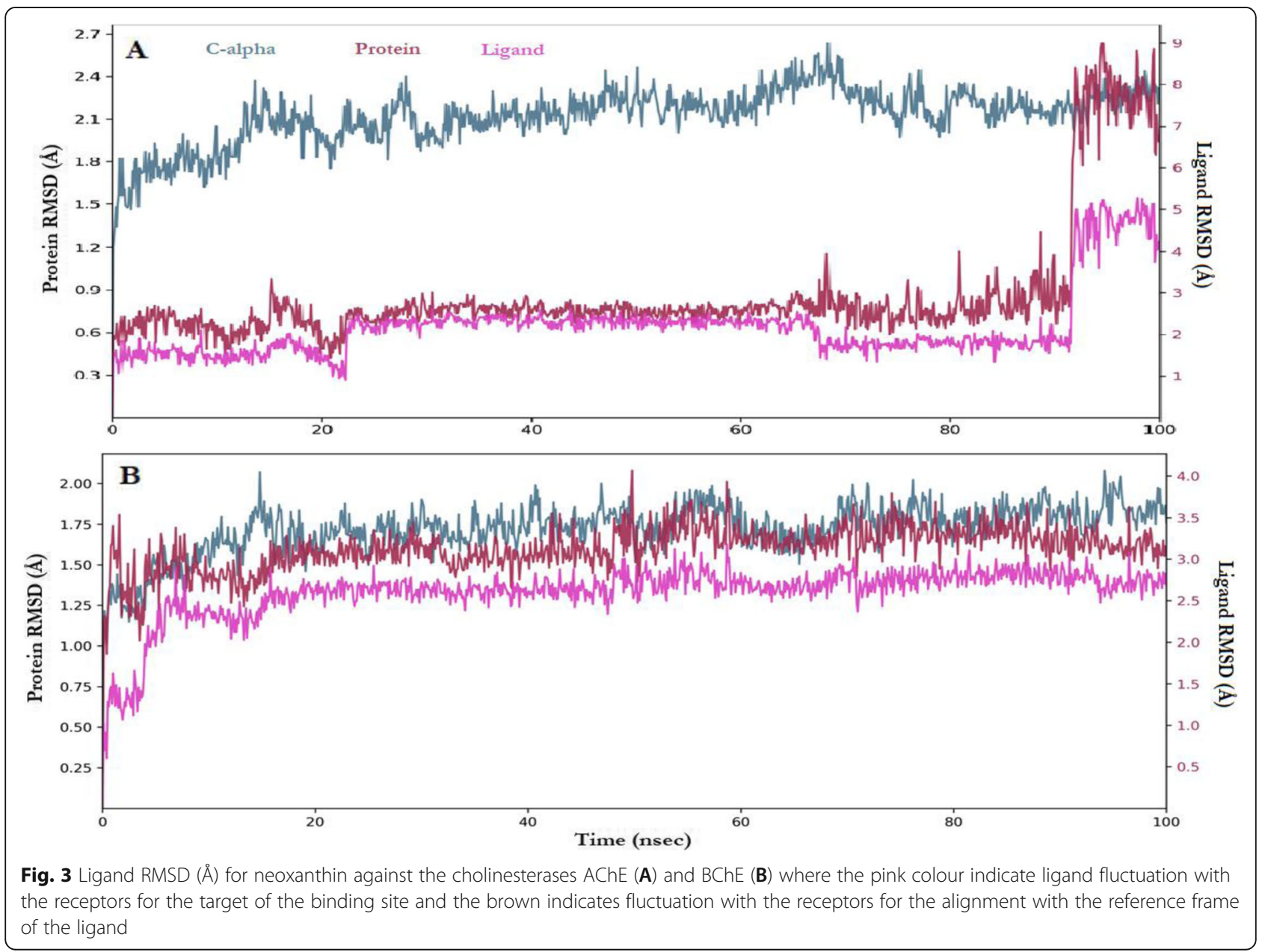



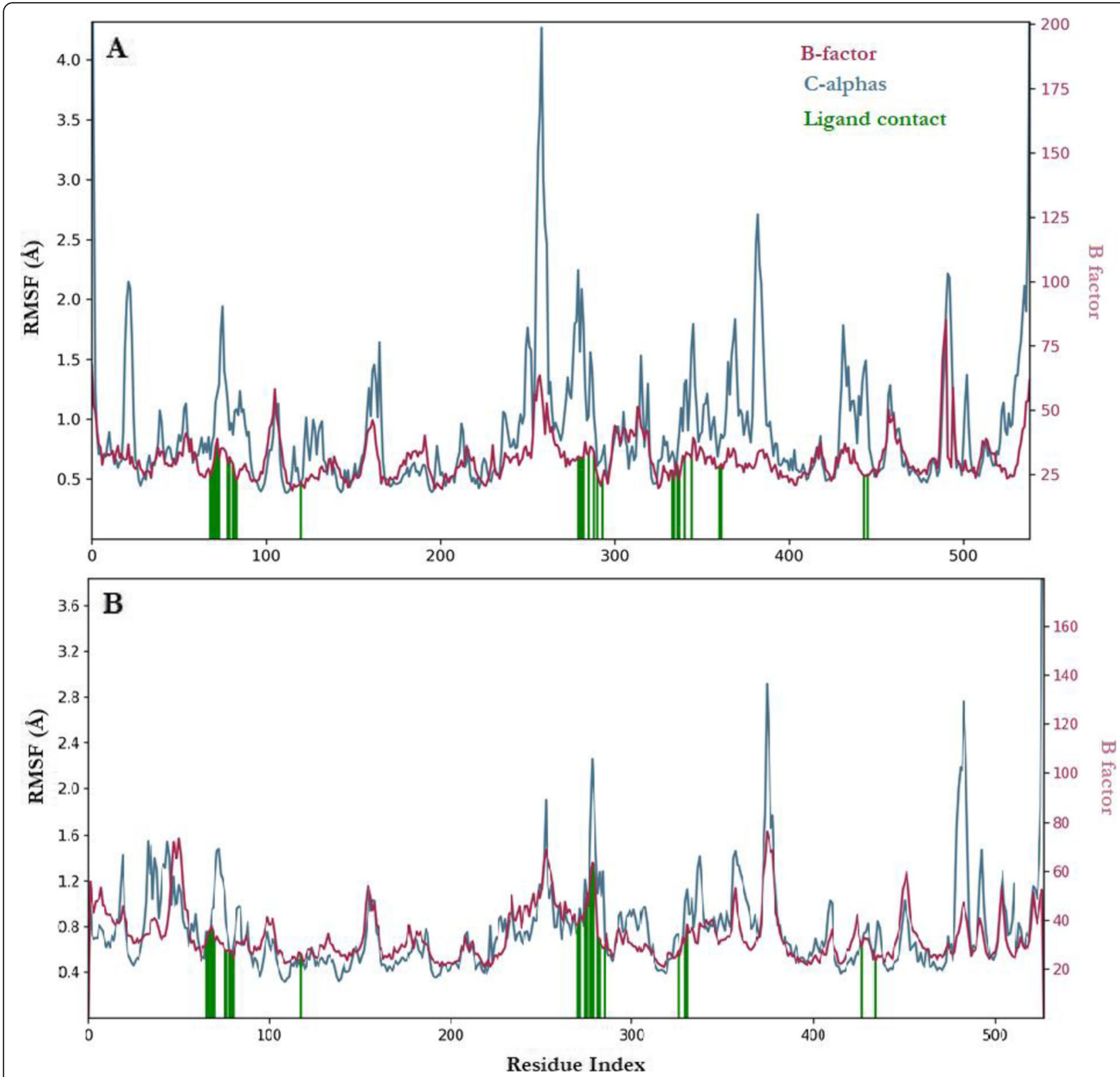

Fig. 4 Protein RMSF interaction with neoxanthin against the cholinesterases AChE $(\mathbf{A})$ and BChE (B) shown in code with green indicating the interaction of neoxanthin atoms with the receptors

interaction was also observed for neoxanthin-AChE at Tyr_72, Asp_74, Asn_87, Tyr_124, Tyr_337, and Tyr 341 with the strongest and most stable ones formed for (Asp_74, Tyr_72, and His_447) whilst neoxanthin-BChE had hydrogen bonding at Asn_68, Asp_70, Gln_71, Ser 72, Gly_78, Ser_79, Trp_82, Ala_328, and Tyr_332 with the strongest and most stable ones formed for (Ile_69, Ser_72, and Tyr_332). Hydrophobic interaction was observed for neoxanthin-AChE complex at Tyr_72, Leu 76, Trp_86, Trp_286, Leu_289, Val_294, Phe_297, Tyr 337, Phe_338, and Tyr_341 whilst neoxanthin-BChE complex had hydrophobic interaction at Met_81, Trp_
82, Ala_277, Phe_278, Val_280, Tyr_282, Pro_285, Phe 329, Tyr_332, Trp_430, and Met_437. Ionic interaction was observed for neoxanthin-BChE complex at Asn_68 and Ile_69 (Table 6).

The interaction of the amino acid residues of the cholinesterases and the neoxanthin, as displayed in the Fig. 6 , was responsible for the overall stability of the ligand using the different bond parameters.

Figure 6 below showed the molecular properties of neoxanthin receptor complexes using RMSD, radius of gyration (rGyr), intramolecular hydrogen bonds (intraHB), molecular surface area (MolSA), solvent 


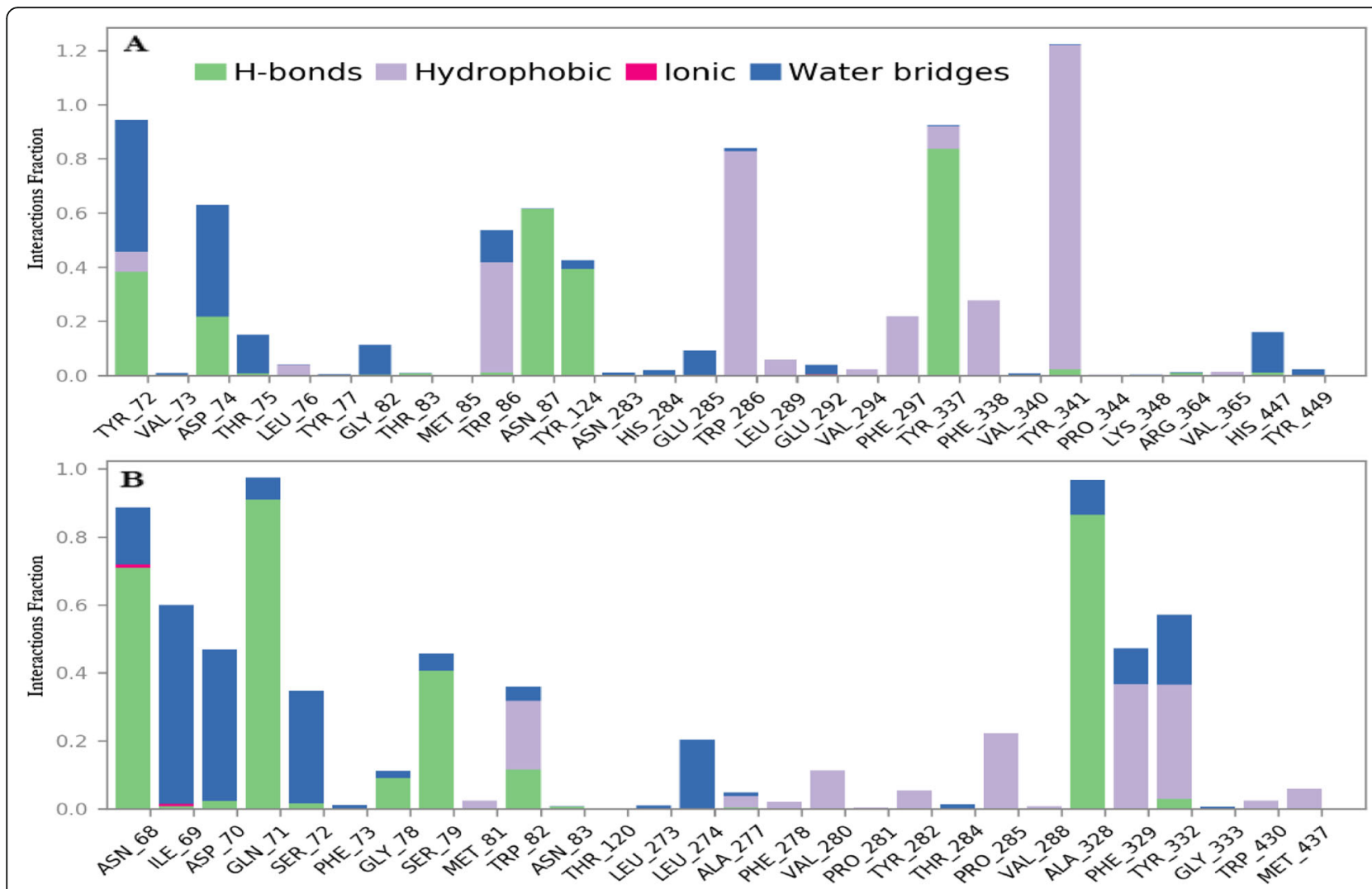

Fig. 5 The histogram chart of interactions of neoxanthin-receptor complexes with AChE (A) and BChE (B) forming hydrogen bond, ionic bond, water bridg, and hydrophobic interactions using MD simulations

accessible surface area (SASA), and polar surface area (PSA) carried out over 100 ns. The RMSD of the ligand indicated fluctuation over time; rGyr indicated neoxanthin extendedness using the principal moment of inertia; intraHB indicated the presence of internal bonds within neoxanthine; MolSA indicated the presence of van der Waal surface area; SASA indicated the presence of surface area accessible by water molecule; and the PSA showed that the solvent accessible surface area contributed by nitrogen and oxygen. In summary, all these properties were present for neoxanthin, indicating no large change was found for neoxanthin-AChE and neoxanthin-BChE.

\section{Discussion}

In an attempt to enhance the management of 'Alzheimer's disease, many researchers have carried out studies to support the therapeutic intervention to manage and treat the disease. One of the major successes is the suppression or inhibition of $\mathrm{AChE}$ and $\mathrm{BChE}$ to delay the breakdown of acetylcholine into the synaptic cleft [41]. In line with the previous study, inhibition of these cholinesterases using carotenoids from ESC and CER has been utilized as an alternative therapy for 'Alzheimer's disease [35]. The knowledge about using natural phytochemicals as drugs is becoming important because of their safety and minor/no side effects [9]. This research, therefore, attempts to test and screen the anticholinesterase efficacy of four carotenoids found in ESC and CER for the treatment of 'Alzheimer's disease. Several carotenoids have been described to inhibit AChE and $\mathrm{BChE}$, traverse the blood-brain barrier and possess antioxidant properties [33, 35]. This extraordinary potential has made them therapeutic candidates against neurodegenerative diseases, most especially 'Alzheimer's disease. With the use of in silico technology, the determination of the best therapeutic agent from the four carotenoid compounds with inhibitory efficacy against cholinesterases could serve as a potential drug with reduced toxicity, using donepezil as an index.

The use of in silico tools for biological analysis is becoming invaluable due to their importance in shortening the formulation period of novel drug candidates for clinical application and the tendency to reduce cost [48]. Appropriate drug target is fundamental to the drug discovery process. In contrast, validation of such a process is necessary to ascertain a level of confidence in the pharmacological potency of the disease [32]. During drug discovery and design of a candidate compound, several intensive tests such as oral bioavailability using 


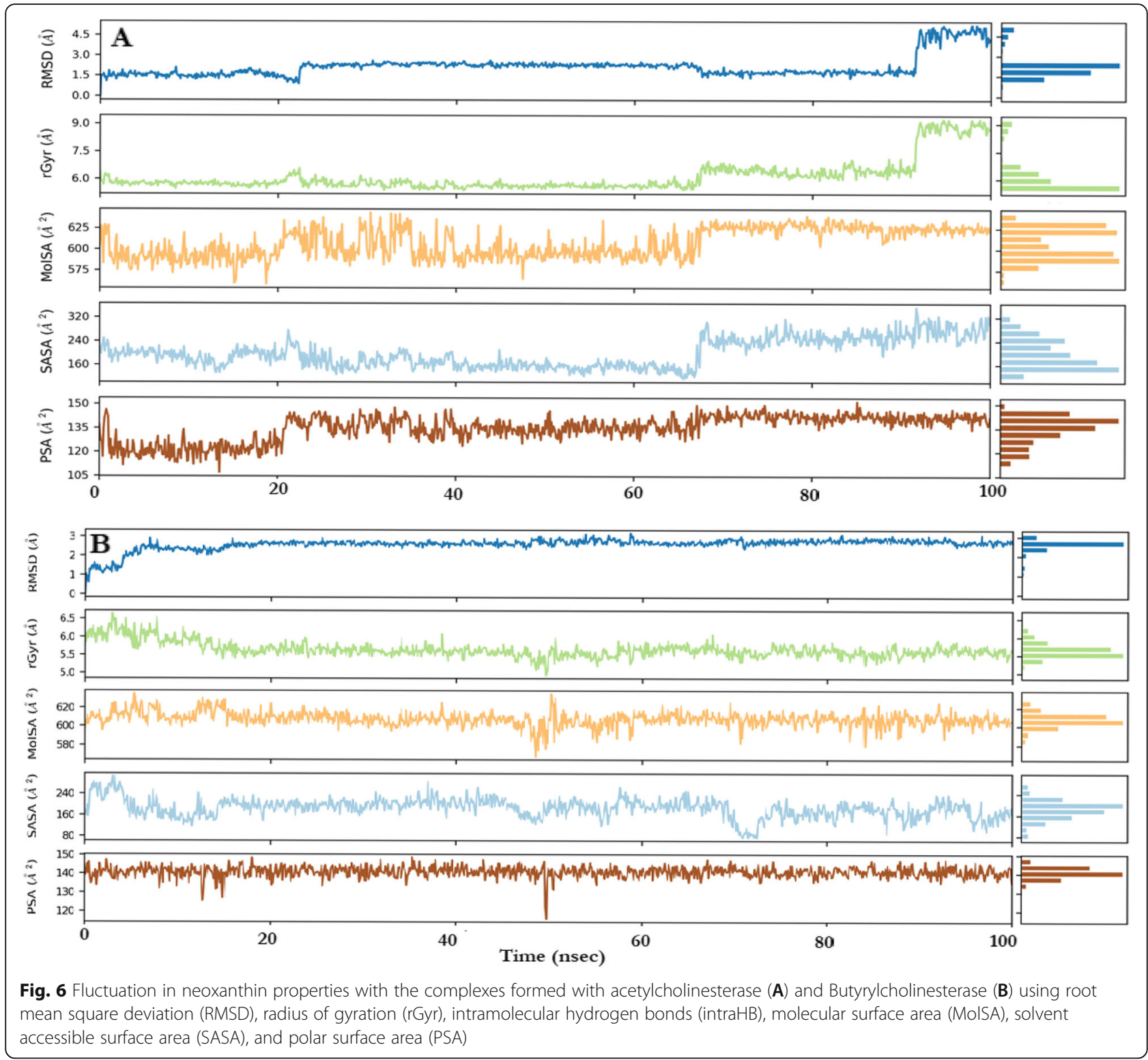

Table 6 Bonding interactions displayed by neoxanthin against Acetylcholinesterase (AChE) and Butyrylcholinesterase (BChE)

\begin{tabular}{|c|c|c|}
\hline Bonding interactins & AChE amino acid residues with positions & BChE amino acid residues with positions \\
\hline $\begin{array}{l}\text { Favoured water bridge } \\
\text { interaction with nitrogen } \\
\text { atom }\end{array}$ & $\begin{array}{l}\text { Tyr_72, Asp_74, Thr_75, Gly_82, Trp_86, Tyr_124, } \\
\text { His_284, Glu_285, Glu_292, and His_447 }\end{array}$ & $\begin{array}{l}\text { Asn_68, Ile_69, Asp_70, Gln_71, Ser_72, Gly_78, Ser_79, Trp_82, } \\
\text { Leu_274, Ala_277, Ala_328, Phe_329, and Tyr_332 }\end{array}$ \\
\hline Hydrogen bonding interaction & $\begin{array}{l}\text { Tyr_72, Asp_74, Asn_87, Tyr_124, Tyr_337, and Tyr_ } \\
341\end{array}$ & $\begin{array}{l}\text { Asn_68, Asp_70, Gln_71, Ser_72, Gly_78, Ser_79, Trp_82, Ala_ } \\
328 \text {, and Tyr_332 }\end{array}$ \\
\hline Hydrophobic interaction & $\begin{array}{l}\text { Tyr_72, Leu_76, Trp_86, Trp_286, Leu_289, Val_294, } \\
\text { Phe_297, Tyr_337, Phe_338, and Tyr_341 }\end{array}$ & $\begin{array}{l}\text { Met_81, Trp_82, Ala_277, Phe_278, Val_280, Tyr_282, Pro_285, } \\
\text { Phe_329, Tyr_332, Trp_430, and Met_437 }\end{array}$ \\
\hline Ionic Interaction & - & Asn_68 and Ile_69 \\
\hline
\end{tabular}


tools such 'Lipinski's rule of five and toxicity test using tools such as PreADMET version 2,0, among others. These facilitate the early advancement in preclinical design and it also helps to avoid clinical failures and reduces cost in the late stage of the preclinical trial [24, 25]. All four compounds (lutein, neoxanthin, violaxanthin, and betacarotene) satisfied only three of 'Lipinski's rule of five with their molecular weight and lipophilicity out of range. It is also interesting to see that even donepezil, a standard anticholinesterase also violates one of the rules. It is believed that with the use of certain biomedical and pharmacological technologies such as nanocomminution, the utilization of high molecular weight and high lipophilic compounds as potent drugs is feasible for stability and bioavailability $[8,10]$.

Apart from this, the toxicity test for the compounds were also carried out in animal models with consideration to their carcinogenic potential; interestingly, these compounds share the same non-toxicity properties with donepezil, a standard drug for the amelioration of the disease. The pharmacological/biological activities of the carotenoids revealed significant blood brain barrier partition coefficient, pure water solubility, skin permeability, human intestinal absorption, plasma protein binding, and buffer solubility, with donepezil scoring lowest in its capacity to cross the blood brain barrier, bind plasma protein, and permeate the skin. These properties are very necessary for central nervous system activities and bioavailability. Lutein was a major concern in its capacity to dissolve in appropriate buffer. This result is in line with the work of Harika et al. [21] where docking study of benzimidazole was carried out using these pharmacological activities. However, it is still important to evaluate the sensitivity test of these carotenoids for their capacity to bind AChE and BChE targets specifically despite their excellent biological activities. For this reason, docking interaction analysis of the five bioactive compounds against $\mathrm{AChE}$ and $\mathrm{BChE}$ (Table 4) using Patchdock revealed that the ligands possessed higher binding affinity than donepezil, with the highest value recorded for beta-carotene. Table 4 showed the area of interaction for the compounds against the cholinesterases with the highest value recorded for beta-carotene. It was also observed that the lowest $\mathrm{ACE}$ values against $\mathrm{AChE}$ and $\mathrm{BChE}$ were recorded for betacarotene. However, the Patchdock result only displayed interaction between ligand and receptor without showing the extent of inhibition of the ligands on the receptors and the site of inhibition [12].

Furthermore, the anticholinesterase efficacy of the ligands was carried out where the molecular docking using the Schrodinger Maestro suit identified the ligandbinding pockets (sites) and conformation of the targets in the receptors using donepezil (a cholinesterase inhibitor used to manage 'Alzheimer's disease) (Table 5). Neoxanthin formed hydrogen bonding with the cholinesterases at three unique locations respectively with the three unique amino acids, whereas donepezil formed three unique bonds for acetylcholinesterase only. This unique property is important for polar activation, ligand affinity, and folding of the receptors, which justified the use of neoxanthin as a cholinesterase inhibitor [43, 49]. Docking analytical study using donepezil and the ligands against the receptors with Schrodinger Maestro suit showed that only donepezil and neoxanthin had appreciable anticholinesterase activities against the receptors with the lowest glide and binding scores observed for donepezil and neoxanthin, respectively. However, the only neoxanthin had a much lower molecular mechanically generalized Born surface area (MM-GBSA) than donepezil. Several studies have reported donepezil as a standard drug for managing 'Alzheimer's disease through cholinesterase inhibition [46]. The high docking energy, glide energy, and molecular mechanically generalized Born surface area (MM-GBSA) scores of neoxanthin could be employed for rational drug discovery in the management of 'Alzheimer's dementia.

Experimental evidence exists to strengthen further the results from this study that carotenoids can reduce the incidence of Alzheimer's disease with oxidative stress in the brain being said to cause a range of problems, including cognitive decline and onset of Alzheimer's disease. A diet high in antioxidants, including vitamin C, vitamin $\mathrm{E}$, and carotenoids such as beta-carotene, may aid in minimizing the incidence of Alzheimer's disease, according to several population studies [22]. There is also evidence that lutein can pass the blood-brain barrier. Multiple studies showed lutein to be strongly linked to age-related cognitive decline and the risk of Alzheimer's disease in humans [51]. However, cholinesterase inhibitors, which improve acetylcholine availability at cholinergic synapses, are currently the main cornerstone therapies for Alzheimer's disease [11]. This study, therefore, explored the anticholinesterase inhibition of lutein, betacarotene, neoxanthine, and violaxanthine for the treatment of Alzheimer's disease. This in silico study corroborated the previous experimental studies that carotenoids can reduce the incidence of cognitive impairment such as Alzheimer's disease, with neoxanthine having better performance as a cholinesterase inhibitor than the other carotenoids used in this study. This is the first study that compared and evaluated the performance of these carotenoids using their anticholinesterase rather than antioxidant properties.

The significance of this study lies in the direction of a fresh route that could help in Alzheimer's treatment development. This is because the knowledge that neoxanthin and other carotenoids could inhibit 
cholinesterases more effectively from a natural source with conformational stability that ensured minimal energy was dissipated throughout the whole system for assurance flexibility towards handling during drug formulation using relevant stereochemistry. The identification of $\mathrm{AChE}$ and $\mathrm{BChE}$ as targets for the carotenoids was very essential for the overall drug development success. From this finding, it could be inferred that neoxanthin would make a good adjuvant for drug formulation against 'Alzheimer's disease using anticholinesterase mechanism, and our result is significant because it is the first in silico study of the bioactive compounds present in Lycopersicon esculentum against $\mathrm{AChE}$ and BChE for the amelioration of 'Alzheimer's disease.

\section{Conclusion}

This research work used in silico technologies to screen several carotenoids in terms of their druggable potency towards inhibition of cholinesterases to ameliorate 'Alzheimer's disease. Despite significant biological/pharmacological potency and safety exhibited by these carotenoids more than donepezil, most especially betacarotene, neoxanthin had specific docking interaction at the binding sites expected for the inhibition of the cholinesterases. The outcomes of this finding showed that neoxanthin could serve as a drug-likeness against cholinesterases in the management of the disease. It is noteworthy to add that neoxanthin can be used in the formulation of diet supplements or as a standalone drug using the technologies used in this research for the intervention of public health concerns caused by the disease. Thus, its utility as an individual oral drug design is safe and efficacious against 'Alzheimer's disease.

\section{Future work}

Future work would incorporate the in vivo and in vitro studies such as cytotoxicity studies and molecular validation assays to further establish the significance of the compounds relative to these in silico studies for corroboration of safety, proper delivery and bioavailability as oral drug candidates. Clinical trials in human beings would follow to establish the molecular dynamics simulation reports.

\section{Acknowledgments}

The authors would like to acknowledge the Department of Biotechnology, University of the Western Cape, to support the accomplishment of the research.

Statement and material availability

All data are available subject to request.

\section{Authors' contributions}

OOB conceived and designed the experiment. OOB, KAA, and AOF carried out the research and drafted the manuscript. MOA, MK, and AK critically evaluated the manuscript. All authors read and approved the final manuscript.

Funding

National Research Foundation, South Africa 120712.

\section{Declarations}

Ethics approval and consent to participate

Not applicable.

Consent for publication

Not applicable.

\section{Competing interests}

The authors declare there is no conflict of interest.

\section{Author details}

${ }^{1}$ Bioinformatics research group, Department of Biotechnology, Faculty of Natural Sciences, University of the Western Cape, Private Bag X17, Bellville, Cape Town 7535, South Africa. ${ }^{2}$ Environmental Biotechnology Laboratory (EBL), Department of Biotechnology, Faculty of Natural Sciences, University of the Western Cape, Cape Town, South Africa. ${ }^{3}$ Department of Science and Technology/Mintek Nanotechnology Innovation Centre, Biolabels Node, Department of Biotechnology, Faculty of Natural Sciences, University of the Western Cape, Private Bag X17, Bellville 7535, South Africa. ${ }^{4}$ Department of Medical Bioscience, University of the Western Cape, Bellville, Cape Town 7535, South Africa. ${ }^{5}$ Plant Omics group, Department of Biotechnology, Faculty of Natural Sciences, University of the Western Cape, Private Bag X17, Bellville, Cape Town 7535, South Africa.

Received: 22 April 2021 Accepted: 8 September 2021

Published online: 01 October 2021

\section{References}

1. Adekiya TA, Aruleba RT, Klein A, Fadaka AO. In silico inhibition of SGTP4 as a therapeutic target for the treatment of schistosomiasis. Journal of Biomolecular Structure and Dynamics. 2020:1-9.

2. Association A. 2018 'Alzheimer's disease facts and figures. 'Alzheimer's \& Dementia. 2018;14:367-429.

3. Biovia DS. Discovery studio visualizer, vol. 936. San Diego; 2017.

4. Birks J. S. and Harvey R. J. 2018. Donepezil for dementia due to 'Alzheimer's disease. Cochrane Database of systematic reviews.

5. Brodaty H, Donkin M. Family caregivers of people with dementia. Dialogues Clin Neurosci. 2009;11:217.

6. Cao J, Hou J, Ping J, Cai D. Advances in developing novel therapeutic strategies for Alzheimer's disease. Mol Neurodegener. 2018;13(1):64. https:// doi.org/10.1186/s13024-018-0299-8.

7. Chaudhari SP, Dave RH. Evaluating the effects of different molecular weights of polymers in stabilizing supersaturated drug solutions and formulations using various methodologies of the model drug: fenofibrate. J Pharm Sci Pharmacol. 2015;2(3):259-76. https://doi.org/10.1166/jpsp.2015.1 066.

8. Choi J-Y, Park CH, Lee J. Effect of polymer molecular weight on nanocomminution of poorly soluble drug. Drug Delivery. 2008;15(5):347-53. https://doi.org/10.1080/10717540802039113.

9. D'Onofrio G, Sancarlo D, Ruan Q, Yu Z, Panza F, Daniele A, et al. Phytochemicals in the treatment of 'Alzheimer's disease: a systematic review. Curr Drug Targets. 2017;18(13):1487-98. https://doi.org/10.2174/13 89450117666161102121553.

10. Ditzinger F, Price DJ, Ilie AR, Köhl NJ, Jankovic S, Tsakiridou G, et al. Lipophilicity and hydrophobicity considerations in bio-enabling oral formulations approaches-a PEARRL review. J Pharm Pharmacol. 2019;71(4): 464-82. https://doi.org/10.1111/jphp.12984.

11. Doraiswamy PM. Non-cholinergic strategies for treating and preventing Alzheimer's disease. CNS drugs. 2002;16(12):811-24. https://doi.org/10.2165/ 00023210-200216120-00003.

12. Duhovny D, Nussinov R, Wolfson HJ. Efficient unbound docking of rigid molecules, International workshop on algorithms in bioinformatics: Springer; 2002. p. $185-200$. 
13. Elger CE, Helmstaedter C, Kurthen M. Chronic epilepsy and cognition. Lancet Neurol. 2004;3(11):663-72. https://doi.org/10.1016/S1474-4422(04 )00906-8.

14. Essman U, Perera L, Berkowitz M, Darden T, Lee H, Pedersen L. A smooth particle mesh ewald potential. J Chem Phys. 1995;103(19):8577-92. https:// doi.org/10.1063/1.470117.

15. Fadaka AO, Aruleba RT, Sibuyi NRS, Klein A, Madiehe AM, Meyer M. Inhibitory potential of repurposed drugs against the SARS-CoV-2 main protease: a computational-aided approach. Journal of Biomolecular Structure and Dynamics. 2020.

16. Fadaka AO, Pretorius A, Klein A. MicroRNA assisted gene regulation in colorectal Cancer. Int J Mol Sci. 2019;20(19):4899. https://doi.org/10.3390/ ijms20194899.

17. Fadaka AO, Sibuyi NRS, Madiehe AM, Meyer M. Computational insight of dexamethasone against potential targets of SARS-CoV-2. Journal of Biomolecular Structure and Dynamics. 2020:1-11.

18. Fadaka AO, Sibuyi NRS, Martin DR, Klein A, Madiehe A, Meyer M. Development of Effective Therapeutic Molecule from Natural Sources against Coronavirus Protease. International journal of molecular sciences. 2021;22(17):9431

19. Ferreira JP, Albuquerque HM, Cardoso SM, Silva AM, Silva VL. Dual-target compounds for Alzheimer's disease: natural and synthetic AChE and BACE-1 dual-inhibitors and their structure-activity relationship (SAR). Eur J Med Chem. 2021;221:113492.

20. Guieu B, Lecoutey C, Legay R, Davis A, Sopkova de Oliveira Santos J, Altomare $C D$, et al. First synthesis of racemic trans Propargylaminodonepezil, a Pleiotrope agent able to both inhibit AChE and MAO-B, with potential interest against Alzheimer's disease. Molecules. 2021;26:80.

21. Harika MS, Kumar TR, Reddy LSS. Docking studies of benzimidazole derivatives using hex 8.0. Int J Pharm Sci Res. 2017;8:1677.

22. Hira S, Saleem U, Anwar F, Sohail MF, Raza Z, Ahmad B. $\beta$-Carotene: a natural compound improves cognitive impairment and oxidative stress in a mouse model of streptozotocin-induced Alzheimer's disease. Biomolecules. 2019;9:441.

23. Jayaram B, Singh T, Mukherjee G, Mathur A, Shekhar S, Shekhar V. Sanjeevini: a freely accessible web-server for target directed lead molecule discovery. BMC bioinformatics. 2012;13(S17):S7. https://doi.org/10.1186/14 71-2105-13-S17-S7.

24. Jin M, Shepardson N, Yang T, Chen G, Walsh D, Selkoe DJ. Soluble amyloid $\beta$-protein dimers isolated from Alzheimer cortex directly induce tau hyperphosphorylation and neuritic degeneration. Proc Natl Acad Sci. 2011; 108:5819-24.

25. Jiraungkoorskul W. Review of neuro-nutrition used as anti-alzheimer plant, spinach, Spinacia oleracea. Pharmacogn Rev. 2016;10(20):105-8. https://doi. org/10.4103/0973-7847.194040

26. Kim S, Thiessen PA, Bolton EE, Chen J, Fu G, Gindulyte A, et al. PubChem substance and compound databases. Nucleic Acids Res. 2016:44(D1):D120213. https://doi.org/10.1093/nar/gkv951.

27. Kinney JW, Bemiller SM, Murtishaw AS, Leisgang AM, Salazar AM, Lamb BT. Inflammation as a central mechanism in 'Alzheimer's disease. 'Alzheimer's Dementia. 2018:4:575-90.

28. Lee S, Lee I, Kim H, Chang G, Chung J, No K. The PreADME approach: webbased program for rapid prediction of physico-chemical, drug absorption and drug-like properties, EuroQSAR designing drugs and crop protectants: processes, problems and solutions; 2003. p. 418-20.

29. Lipinski CA. Lead-and drug-like compounds: the rule-of-five revolution. Drug Discov Today Technol. 2004;1(4):337-41. https://doi.org/10.1016/j.ddtec.2 004.11.007.

30. Magnus $\mathrm{G}$. The age of aging: how demographics are changing the global economy and our world: Wiley; 2012. https://doi.org/10.1002/97811192 07702.

31. Makhoba XH, Viegas C Jr, Mosa RA, Viegas FP, Pooe OJ. Potential impact of the multi-target drug approach in the treatment of some complex diseases. Drug Des Devel Ther. 2020;14:3235-49. https://doi.org/10.2147/DDDT.S257494.

32. Moffat JG, Vincent F, Lee JA, Eder J, Prunotto M. Opportunities and challenges in phenotypic drug discovery: an industry perspective. Nat Rev Drug Discov. 2017;16(8):531-43. https://doi.org/10.1038/nrd.2017.111.

33. Moyo M, Ndhlala AR, Finnie JF, Van Staden J. Phenolic composition, antioxidant and acetylcholinesterase inhibitory activities of Sclerocarya birrea and Harpephyllum caffrum (Anacardiaceae) extracts. Food Chem. 2010;123(1):69-76. https://doi.org/10.1016/j.foodchem.2010.03.130.
34. Muller S, Brun S, René F, de Sèze J, Loeffler J-P, Jeltsch-David H. Autophagy in neuroinflammatory diseases. Autoimmun Rev. 2017;16(8):856-74. https:// doi.org/10.1016/j.autrev.2017.05.015.

35. Oboh G, Bakare O, Ademosun A, Akinyemi A, Olasehinde T. Inhibition of cholinesterases and some pro-oxidant induced oxidative stress in rats brain by two tomato (Lycopersicon esculentum) varieties. Int J Biomed Sci. 2015;11:48.

36. Ojo OA, Aruleba RT, Adekiya TA, Sibuyi NRS, Ojo AB, Ajiboye BO, et al. Deciphering the interaction of puerarin with cancer macromolecules: an in silico investigation. J Biomol Struct Dyn. 2020:1-12. https://doi.org/10.1080/ 07391102.2020.1819425.

37. Omondi RO, Sibuyi NR, Fadaka AO, Meyer M, Jaganyi D, Ojwach SO. Role of $\pi$-conjugation on the coordination behaviour, substitution kinetics, DNA/ BSA interactions, and in vitro cytotoxicity of carboxamide palladium (ii) complexes. Dalton Transactions. 2021

38. Oyinloye BE, Adekiya TA, Aruleba RT, Ojo OA, Ajiboye BO. Structure-based docking studies of GLUT4 towards exploring selected phytochemicals from Solanum xanthocarpum as a therapeutic target for the treatment of Cancer. Curr Drug Discovery Technol. 2019;16(4):406-16. https://doi.org/10.2174/1 570163815666180801152110

39. Padron J, Carrasco R, Pellon R. Molecular descriptor based on a molar refractivity partition using Randic-type graph-theoretical invariant. J Pharm Pharmaceut Sci. 2002:5:258-66.

40. Radhakrishnan K. Challenges in the management of epilepsy in resourcepoor countries. Nat Rev Neurol. 2009;5(6):323-30. https://doi.org/10.1038/ nrneurol.2009.53.

41. Rao AA, Sridhar GR, Das UN. Elevated butyrylcholinesterase and acetylcholinesterase may predict the development of type 2 diabetes mellitus and Alzheimer's disease. Med Hypotheses. 2007;69(6):1272-6. https://doi.org/10.1016/j.mehy.2007.03.032.

42. Rossi M, Freschi M, de Camargo Nascente L, Salerno A, de Melo Viana Teixeira S, Nachon F, et al. Sustainable drug discovery of multi-targetdirected ligands for Alzheimer's disease. J Med Chem. 2021;64(8):4972-90. https://doi.org/10.1021/acs.jmedchem.1c00048.

43. Scheer A, Fanelli F, Costa T, De Benedetti P, Cotecchia S. Constitutively active mutants of the alpha $1 \mathrm{~B}$-adrenergic receptor: role of highly conserved polar amino acids in receptor activation. EMBO J. 1996;15(14): 3566-78. https://doi.org/10.1002/j.1460-2075.1996.tb00726.x.

44. Schneidman-Duhovny D, Inbar Y, Nussinov R, Wolfson HJ. PatchDock and SymmDock: servers for rigid and symmetric docking. Nucleic Acids Res. 2005;33(Web Server):W363-7. https://doi.org/10.1093/nar/gki481.

45. Selkoe DJ. Biochemistry and molecular biology of amyloid $\beta$-protein and the mechanism of 'Alzheimer's disease. Handb Clin Neurol. 2008;89:245-60. https://doi.org/10.1016/S0072-9752(07)01223-7.

46. Tsuno N. Donepezil in the treatment of patients with Alzheimer's disease Expert Rev Neurother. 2009;9(5):591-8. https://doi.org/10.1586/ern.09.23.

47. Uddin MS, Al MA, Kabir MT, Ashraf GM, Bin-Jumah MN, Abdel-Daim MM Multi-target drug candidates for multifactorial Alzheimer's disease: AChE and NMDAR as molecular targets. Mol Neurobiol. 2021;58(1):281-303. https://doi.org/10.1007/s12035-020-02116-9.

48. Wadood A, Ahmed N, Shah L, Ahmad A, Hassan H, Shams S. In-silico drug design: an approach which revolutionarised the drug discovery process. OA Drug Design Delivery. 2013;1:3.

49. Wootten D, Reynolds CA, Smith KJ, Mobarec JC, Furness SG, Miller LJ, et al. Key interactions by conserved polar amino acids located at the transmembrane helical boundaries in class B GPCRs modulate activation, effector specificity and biased signalling in the glucagon-like peptide-1 receptor. Biochem Pharmacol. 2016;118:68-87. https://doi.org/10.1016/j.bcp.2016.08.015.

50. Wu J, Pistolozzi M, Liu S, Tan W. Design, synthesis and biological evaluation of novel carbamates as potential inhibitors of acetylcholinesterase and butyrylcholinesterase. Bioorg Med Chem. 2020;28(5):115324. https://doi. org/10.1016/j.bmc.2020.115324.

51. Xu X, Lin X. Advances in the researches of lutein and 'alzheimer's disease. Zhonghua yu Fang yi xue za zhi [Chinese Journal of Preventive Medicine]. 2015:49(5):456-60.

52. Yunta M. It is important to compute intramolecular hydrogen bonding in drug design. Am J Model Optimization. 2017;5(1):24-57. https://doi.org/1 0.12691/ajmo-5-1-3.

\section{Publisher's Note}

Springer Nature remains neutral with regard to jurisdictional claims in published maps and institutional affiliations. 